ten zu können, sollte die jüngere Juristengeneration endlich dazu bewegen, die politisch-gesellschaftlichen Bedingungen und die geistigen Voraussetzungen für eine solche Kontinuität zu untersuchen. Herr $E$. ist nicht der einzige unter den ehemaligen Mitläufern, der weiterhin versucht, seine Erkenntnisse von damals zu verbreiten -..-

Dieser Beitrag hat eine eigene Geschichte. Er wurde der $»$ Zeitschrift für Rechtspolitik* angeboten. Die Redaktion lehnte ab: "Es ist... die Art und Weise, wie Sie in Ihrer Glosse gegen die Verfasser des erwähnten Loseblatt-Kommentares (ob zu Recht oder zu Unrecht, mag hier dahinstehen) zu Felde ziehen, die uns von einer Veröffentlichung abhält.«

Der Verfasser bat daraufhin die Redaktion um die Erlaubnis, den diese Begründung enthaltenden Brief zusammen mit dem Beitrag in einer anderen juristischen Zeitschrift veröffentlichen zu dürfen: "Diese Begründung har, wie mir scheint, dokumentarischen Wert. Sie zeigt, welche Schwierigkeiten es heute noch (nach über 25 Jahren) bereitet, in welcher Form auch immer die geistige Kumpanei eines Juristen mit dem Nationalsozialismus beispielhaft aufzudecken und auf die Kontinuität seines Denkens bis in die jüngste Zeit hinzuweisen.«

Die Antwort darauf lautete: "Gegen die Aufdeckung von Nachwirkungen nationalsozialistischer Denkweise ins gegenwärtige Rechtsdenken hinein bestünden keinerlei Bedenken. Dafür gibt es auch einen breiten Spielraum von möglichen Formen - geistvoll oder nachdenklich, auch Polemik ist für uns nicht tabu.\&

Roderich Wabsner

\title{
Die Verfassung der Föderation Arabischer Republiken: Ein Exempel supranationaler Notstandsgesetzgebung
}

Am 17. April r 97 I unterzeichneten die Staatschefs Syriens, Libyens und Ägyptens in Bengasi (Libyen) die Gründungsdeklaration der Föderation Arabischer Republiken (FAR). Darin wird hervorgehoben, daß die Föderation die Verwirklichung der Tripoli-Charta vom Dezember 1969 darstelle, die seinerzeit auf Anregung des damals noch wirkenden Nasser entstand und die eine enge Kooperation $\mathrm{z}$ wischen den drei genannten Ländern und darüber hinaus dem Sudan vorsah. Diese Blockbildung läßt sich adäquat nur im Kontext der politischen Entwicklung in den arabischen Ländern seit 1967 begreifen.

Bekanntlich war die Niederlage der arabischen Staaten im Juni-Krieg 1967 der Anlaß einer tiefgreifenden innenpolitischen Krise in allen betroffenen Ländern. Die Armee, Träger der politischen Macht in diesen Ländern, wurde durch die Niederlage entblößt. Fortan konnten die arabischen Armeen ihre Herrschaft nicht mehr legitimieren, indem sie sich als Träger der nationalen und sozialen Revolution propagierten. Die Niederlage hatte den Tatbestand transparent gemacht, auf den die Kritiker der arabischen Militärdiktaturen schon lange zuvor hingewiesen hatten ${ }^{1}$, daß nämlich die Armee, die den zivilen großbürgerlichen Regierungen die politische Macht entrissen hatte, um die Korruption zu beseiti-

1 Cf, z. B. A. Abdel-Malek, Agypten: Militärgesellschaft. Das Armeeregime, die Linke und der soziale Wandel unter Nasser. Frankfurt/M. I971 (Orig. franz. I962), u. a. 
gen und um eine umfassende soziale Transformation und Emanzipation von den imperialistischen Abhängigkeitsverhältnissen zu ermöglichen, bei allem Anspruch nach ihrer Machtergreifung die alten Strukturen mit einigen Modifikationen beibehielt und die Privilegien der bisher Herrschenden nun für sich in

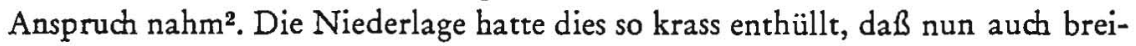
te Bevölkerungsteile die Herrschaftslegitimität des Militärs anzweifelten, wodurch freilich die Arbeit alternativer Bewegungen erleichtert wurde. Innerhalb kurzer Zeit konnten die arabische Linke ${ }^{3}$, eine bislang isolierte, kleine Minderheit, und die von allen Regimes diskriminierten und verfolgten palästinensischen Flüchtlinge, nunmehr zu einer Résistance-Bewegung avanciert ${ }^{4}$, Einfluß und Popularität unter den Massen und der bis dahin zum größten Teil panarabisch-nationalistisch orientierten Intelligenz gewinnen. Die Propaganda und die Demagogie der arabischen Militärdiktaturen nasseristischer und ba'thistischer Observanz wurden jetzt gemeinhin als solche identifiziert. Die militärischen Nachzügler Ghadhafi und Numairi hatten nicht mehr jene günstigen Bedingungen wie einst die "Freien Offiziere in Ägypten und die diversen ba'thistischen Putschofffiziere in Syrien und dem Irak.

Von 1967 und bis Ende 1969 befanden sich die Militärdiktaturen in den arabischen Ländern in der Defensive. Seit der Unterzeichnung der Tripoli-Charta im Dezember 1969 gingen sie zur Offensive über, ohne jedoch zunächst viel Erfolg ernten zu können, denn die palästinensische Résistance war damals noch auf der Höhe ihrer Entwicklung und war noch in der Lage, diesen Regimen ihre Bedingungen zu diktieren.

Die Machtkonstellation änderte sich mit dem Massaker vom September 1970 in Jordanien. Die zahlreichen Fehler, die die Résistance und die arabische Linke begangen hatten, ermöglichten nun eine breit angelegte Offensive, an der sowohl die alten Monarchisten als auch die sich immer noch als »revolutionär « propagierenden arabischen Militärdiktaturen beteiligt waren. Die nach dem Massaker in Jordanien unter dem Vorsitz des ehemaligen tunesischen Ministerpräsidenten Bahi Ladgam ${ }^{5}$ eingesetzte allarabische Schiedsrichterkommission hatte als Mitglieder nachweislich profilierte CIA-Agenten, die als Armee-Offiziere in Burgibas Regime wirkten ${ }^{6}$. Diese Kommission betrieb die politische Neutralisierung der Résistance, nachdem die Bewegung militärisch bereits durch die jordanische Beduinen-Armee geschlagen worden war.

Einige Monate nach dem Seprember-Massaker in Jordanien, dem etwa zehntausend Mann zum Opfer fielen, erschien die Situation für die Gründung der in der Tripoli-Charta vorgesehenen Föderation günstig: In Syrien fand kurz nach

2 Cf. B. Tibi, Zum Verhältnis von Militär und kolonialem Nationalismus am Beispiel der arabischen Länder*, in: Sozialistische Politik, Bd. I (1969), H. 4, pp. 4-19, sowie B. Tibi, -Armee und sozialer Wandel in Nordafrika *, in: Afrika Spectrum, Bd.6 (197I), H. 1, pp.

3 Cf. B. Tibi (ed.), Die arabische Linke, Frankfurt/M. 1969, sowie M. Kischli, Kapitalismus und Linke im Libanon, ed. B. Tibi, Frankfurt/M. 1970.

- Cf. G. Chaliand, La Résistance Paléstinienne, Paris r970; dt. Obers. G. Chaliand, Kann Israel noch besiegt werden, oder: Die Kommandos kämpfen weiter. Reinbek I 97 r. Cf. auch meine Bemerkungen in: Das Argument, Bd. I2 (1970), H. 59, pp. 624-629.

5 Ladgam gehört zum großbürgerlichen Flügel der tunesischen Neo-Destour-Partei. Ober ihn cf. B. Tibi, $\rightarrow$ Die Krise des Burgibismus. Entstehung und Verfall des ,konstitutionellen Sozialismuss in Tunesien, in: Das Argument, Bd. I2 (1970), H. 59, pp. 530-556.

- Obwohl die tunesische Opposition die palästinensische Résistance darauf aufmerksam gemadut hat, hat diese, besonders al-Fatah, mit der sog. Schiedsrichterkommission verhandelt und zusammengearbeitet. Das offene Schreiben der tunesischen Opposition hierzu ist abgedrudke in: Ibrahim Tubal, Ma'sat Ahmad Ben Salah (Die Tragödie des Ahmed Ben Salah), Beirut r970, Pp. Is4-I74. 
dem Massaker ein Putsch statt, bei dem der rechte Flügel der syrischen Ba'thPartei den als »links« geltenden regierenden Flügel absetzte; in Libyen waren die gewerkschaftliche Bewegung und die studentische Opposition bereits zerschlagen; und in Agypten kamen nach Nassers Tod dessen Erben zu einem $\gg$ Machtausgleich « mit Sadat als Scheinpräsidenten ${ }^{7}$. Allein im Sudan existierte immer noch eine starke und bestens organisierte, dazu relativ souveräne KP, die im Gegensatz zu den anderen arabischen prosowjetischen kommunistischen Parteien, die irakische ausgenommen, keine marginale Größe war und die dem Numairi-Regime die Stirn bot, zumal sie sich auf mobile Organisationen berufen konnte: die Gewerkschaften, die Studentenverbände, die Frauenverbände und die Landarbeiterorganisationen. Es versteht sich daher, warum der Sudan zunächst der Föderation nicht beitreten konnte. Die Bengasi-Gründungsdeklaration sagt über das Fernbleiben des Sudan folgendes aus: "Die drei Gründungspräsidenten betonen, daß die Demokratische Republik Sudan und ihr unter der Führung des Bruders von Präsident Numairi und mit seinen Brüdern kämpfendes arabisches Volk aktiv an der Ausarbeitung der Tripoli-Charta mitwirkten; der Sudan wird auch weiterhin aktiv am panarabischen Kampf partizipieren und enge Bindungen zur Föderation Arabischer Republiken unterhalten, bis er der Föderation als Vollmitglied beitreten kann. ${ }^{8}$

Obwohl der Sudan der Föderation bislang noch nicht beigetreten ist, scheint man sich stillschweigend darauf geeinigt $\mathrm{zu}$ haben, daß die essentiellen Bestimmungen der Föderation, d. h. jene mit Notstandscharakter, auch für den Sudan gelten. Schon in den Leitsätzen heißt es (in $₫ 7, A b s$. d): Zu den Aufgaben der Föderation gehört »die Aufrechterhaltung der nationalen Sicherheit der Unionsstaaten gemäß ihrer Verfassung. Wenn in einer der Föderationsrepubliken von innen oder von außen verursachte Unruhen zustande kommen, die die Sicherheit der Föderation gefährden, wird die Zentralinstanz der Föderation davon in Kenntnis gesetzt, um Maßnahmen innerhalb ihrer Kompetenzen für die Wiederherstellung von Ruhe und Ordnung zu ergreifen. Sollte eine der Föderationsregierungen nicht in der Lage sein, diese Hilfe zu erbitten, dann ist die Föderation befugt, auch obne Aufforderung zu intervenieren, um Ruhe und Ordnung wiederberzustellen. " $^{9}$ Es hat nicht sehr lange gedauert, bis dieser Paragraph zur Anwendung kam. Nach den unermüdlichen, gleichwohl erfolglosen Versuchen des sudanesischen Numairi-Regimes, die KP des Sudan dazu bewegen, sich selbst aufzulösen, um sich sodann nach ägyptischem Muster in eine dem Regime unterstellte Einheitspartei zu integrieren, griff das Numairi-Regime zur Methode des physischen Terrors gegen die Opposition und die mit ihr sympathisierenden Teile der Armee. Allerdings kam es bereits am I9. Juli I97 I zu einem Coup d'état gegen das Terror-Regime, der von linksnationalistischen Offizieren angeführt und von der KP des Sudan unterstützt wurde. Der Putsch verlief bekanntlich erfolgreich. Die verbotenen Suborganisationen der KP wurden sofort wieder zugelassen; im ganzen Sudan erfolgte ein allgemeines Aufatmen, besonders auch deshalb, weil die neuen Machthaber und die KP des Sudan versprachen, der

\footnotetext{
7 Dieser $>$ Machtausgleich $*$ dauerte indes nicht lange. Denn Sadat hat mit der Gründung der FAR versucht, die ihm aufgezwungene Rolle des Scheinpräsidenten loszuwerden und die Macht, die Nasser einst innehatte, zu erlangen, wobei es im Mai 1971 zum offenen Konflikt kam, den Sadat allerdings durch Koalition mit den Armee-Generälen zu seinen Gunsten löste. Cf. dazu B. Tibi, *Nassers Erbe in Agypten*, in: links, 197x, H. 24, pp. 2 1-22.

8 Alle Dokumente der FAR sind abgedrudkt in der Damaszener Zeitschrift *al-Ma'rifa * (x971), H. Irs, pp. 183-205, wonad hier zitiert wird. Eigene Ubersetzung aus dem Arabischen; eigene Hervorhebung.

- Ibid., p. 186.
} 
unterdrückten negroiden Bevölkerung des Südsudan Emanzipationsrechte zu gewähren. Jedoch konnte sich das neue Regime nur vier Tage lang am Leben halten, denn die drei Staaten der FAR brachten jenen schon zitierten Notstandsparagraphen zur Anwendung. Die ansonsten wenig erfolgreiche ägyptische Luftwaffe wurde voll eingesetzt, um Numairi-loyale sudanesische Kadetten aus den ägyptischen Militärakademien und die am Suez-Kanal stationierten sudanesischen Truppen nach dem Sudan zu transportieren. Die libysche Luftwaffe exerzierte Luftpiraterie und kaperte ein britisches Linienflugzeug, das sudanesische Exilpolitiker an Bord hatte, die auf dem Weg nach Khartum waren, um dort Regierungsposten zu übernehmen. Der ägyptische Geheimdienst, der Numairi schon zuvor behilflich gewesen war, tat über seine Agenturen im Sudan alles in seinen Kräften Liegende, um die Etablierung des neuen sudanesischen Regimes zu verhindern. Kurzum: es gelang den Föderationsstaaten, im Sudan »Ruhe und Ordnung wiederherzustellen. «10 Die Putschoffiziere und die Führer der KP des Sudan wurden hingerichtet. Eine breit angelegte antikommunistische Verfolgungsjagd schloß sich an.

In der Tat: die FAR, die nach der Gründungsdeklaration von Bengasi »mit Gottes Hilfe und im Vertrauen der Gläubigen auf Gott im Hinblick auf die Zukunft« installiert worden war, ist bereits »mit Zähnen geboren « worden, wie Sadat, der Präsident der Föderation, anspielend auf die Machtkämpfe in seinem eigenen Land (cf. Anm. 7) und auf die Sudan-Ereignisse, sagte. ${ }^{11}$

Für das Verständnis der neuen Notstandsföderation sind außer der BengasiGründungsdeklaration und den Leitsätzen (dem Gründungsdokument) noch die Damaskus-Deklaration und der sie begleitende "Verfassungsentwurf « der Föderation relevant. Uber den Verfassungsentwurf erfolgte am I. September 1971 eine Volksabstimmung, die - wie üblich - $" 99 \%$ Ja-Stimmen « erbrachte. Seitdem besteht die FAR offiziell als Einheit, obwohl ihre Mitglieder dadurch ihren völkerrechtlichen Charakter nicht verloren haben. Die Verfassung der FAR ist lediglich eine Erweiterung der schon zitierten Leitsätze, die parallel zur BengasiDeklaration formuliert wurden. Dagegen ist die Damaskus-Deklaration eine Sammlung propagandistischer Vokabeln aus der panarabisch-nationalistischen Ideologie, mit denen die Gründung der FAR legitimiert werden soll.12

Die Verfassung der FAR besteht aus mehreren Abschnitten, die im folgenden kurz referiert werden:

Der erste Teil steckt den allgemeinen rechtlichen Rahmen der Föderation ab. Hiernach gehört die Souveränität dem Volk $(\$ 2)$; die arabische Sprache ist die offizielle Verkehrssprache $(\$ \varsigma)$ etc. Es ist interessant, aus $\$$ I 2 zu erfahren, daß die Föderationsrepubliken zwar ihre eigenen Verfassungen haben dürfen, daß diese aber sämtliche Grundrechte, die detailliert angeführt werden (Rede- und Meinungsfreiheit, Versammlungsrecht, Briefgeheimnis, Pressefreiheit etc.) garantieren müssen. Dies erscheint umso zynischer, als offenbar ist, daß in allen Föderationsrepubliken keinerlei Grundrechte praktiziert werden können, ja noch nicht einmal in unterhöhlter Form, wie dies in bürgerlich-demokratischen Ländern der Fall ist. Von Pressefreiheit in einem Land zu sprechen, wo jeder, der öffentlich ein kritisches Wort wagt, seine persönliche Freiheit einbüßt, ist blanker

10 Schon vor den Ereignissen im Sudan wurde dieser Notstandscharakter der FAR im Hinblids auf die politische Lage im Sudan hervorgehoben von B. Tibi, Union Arabischer Republiken a, in: Afrika heute, 1971, H. 10, pp. 199-201.

11 P. Fridse, $\rightarrow$ Zähne im Sudan a, in: Afrika heute, $197 \mathrm{I}, \mathrm{H} .22$, pp. $34 \mathrm{I}-342$.

12 Zur Ideologie des Panarabismus cf. B. Tibi, Nationalismus in der Dritten Welt am arabischen Beispiel, Frankfurt/M. 1971. 
Hohn. Weniger bodenlos ist die Aussage von $\ 6:$ :Die Föderation stützt sich auf spirituelle Werte und nimmt das islamische Recht (die Schari'a ${ }^{13}$ ) zur Quelle seiner Rechtssprechung. « Dieser Paragraph hat seinen besonderen Stellenwert im Zusammenhang mit den Versuchen des ägyptischen Präsidenten Sadat und des libyschen Präsidenten Ghadhafi, die islamische Religion zur Grundlage einer integrativen Politik zu machen. Diese Ideologie erweist sich freilich in Ländern, deren Bevölkerung emotional-gläubig ist, als probates Mittel zur Verketzerung und Verfolgung der linken Opposition. Der Hinweis auf die "gottlosen Kommunisten « mobilisiert Gefühle gegen jene, die über die tatsächlichen Verhältnisse aufklären wollen, und versperrt den mithilfe religiöser Obskurantismen Gegängelten vorab die Einsicht in die eigene Klassenlage. In der neuen ägyptischen Verfassung, die kurz nach der Verfassung der FAR verabschiedet wurde (am Ir. September 1971), wird diesen Mechanismen voll Rechnung getragen. Sie können in Agypten zu besonderer Wirkung kommen, weil die meisten ägyptischen Marxisten sich aus der christlichen Bevölkerung rekrutieren ${ }^{14}$.

Der zweite Teil der Föderationsverfassung befaßt sich mit den Kompetenzen der FAR, soweit die Außenpolitik, die Verteidigung und die "nationale Sicherheit « in Frage stehen. In $\llbracket x_{4}$ heißt es: Der FAR obliegen »der Schutz der nationalen Sicherheit und die Entfaltung eines Planes für die Sicherheit der Föderation gemäß den Bestimmungen des Präsidialrates«, ein Paragraph, der praktisch sämtliche Notstandsmaßnahmen ermöglicht. Ferner gehören die ökonomische Koordination und die Bildungspolitik der Mitgliedsstaaten zu den Kompetenzbereichen der Föderation.

Die Exekutive der FAR umfaßt den Präsidialrat und das Föderationskabinett. Der Präsidialrat konstituiert sich aus dem Präsidenten der Föderationsrepubliken. Inzwischen ist Sadat für zwei Jahre zum Präsidenten dieses Rates gewählt worden. Das Föderationskabinett, der zweite Teil der Exekutive, ist dem Präsidialrat verantwortlich. Auch das Kabinett ist inzwischen gebildet worden. Nominiert wurden der Syrer Ahmad Khatib als Ministerpräsident sowie sieben ägyptische, zwei libysche und ein syrischer Minister.

Die Legislative besteht aus einer 60 Personen umfassenden Nationalversammlung, wobei je 20 Abgeordnete aus einer der Föderationsrepubliken kommen, wo sie aus den jeweiligen "Volksvertretungen" ausgewähit wurden.

Die Judikative ist in einem Bundesverfassungsgericht verkörpert, dem sechs Richter angehören, je zwei aus jedem Mitgliedsstaat, die zusammen vom Präsidialrat nominiert werden. Während die Rechtsurteile der nationalen Gerichte jeweils nur auf den Geltungsbereich einer einzelnen Föderationsrepublik beschränkt bleiben, sind die Urteile des Bundesverfassungsgerichts in allen drei Föderationsrepubliken rechtskrättig.

Die Verfassung der FAR enthält neben den referierten Teilen einen Abschnitt über das Föderationsbudget und einen abschließenden Teil mit "Ubergangsbestimmungen «. In diesem letzten Teil stößt man wiederum auf in der Aussage interessante Paragraphen, die nicht - wie die meisten Paragraphen der Verfassung - juristische Ausschmückungen enthalten, die aus westlichen Ländern importiert wurden und der Wirklichkeit in keiner Weise entsprechen. Am wichtigsten erscheint der $\$ 64$, der wortwörtlich mit dem bereits vollständig zitierten

13 Zur islamischen Rechtsprechung cf. H. A. R. Gibb, Mohammedanism, London $1965^{2}$, pp. 88-ro6, sowie M. Khadduri, s The Nature and Sources of the Shari'ae, in: The George Washington Law Review, Bd. 22 (1953), pp. 3-23.

${ }_{14}$ Cf. hierüber jetzt Die Lage der Kopten im Agypten Sadats «, in: NZZ vom 30. Dez. 1971, Bl. 3; dieser Artikel gibt Aufschluß über die Islamisierungspolitik Sadats. 
$\$ 7, A b s . d$, aus den Leitsätzen der Föderation (Gründungsdokument) übereinstimmt. Er gestattet den einzelnen Föderationsrepubliken die Intervention in jenen Föderationsteilen, in denen "innenpolitische Unruhen « stattfinden, d. h. also, daß künftig jede oppositionelle Bewegung, die eine Machteroberung anstrebt, nicht nur mit den lokalen Polizei- und Armee-Apparaturen konfrontiert ist, sondern auch mit jenen der übrigen Föderationsrepubliken. Der Inhalt dieses Paragraphen gibt der FAR ihren Grundcharakter. Es ist sehr zweifelhaft, ob es zu einer alle wichtigen sozialen Bereiche umfassenden Föderation kommen wird. In den Ubergangsbestimmungen ist deutlich hervorgehoben, daß die Mitgliedschaft in der FAR den Völkerrechtscharakter der Mitglieder nicht aufhebt; daß die einzelnen Republiken also ihre Angelegenheiten selbst regeln $(\$ 60-61)$. Die Leitsätze der Föderation $\left(\mathbb{S} \mathrm{r}_{3}\right)$ verbieten auch den in einer der Republik wirkenden politischen Organisationen die Arbeit in den restlichen Teilen der Föderation. So darf die syrische Ba'th-Partei nicht in Agypten oder gar in Libyen agitieren. Dieser Paragraph der Leitsätze ist enthalten in $\$ 62$ der Verfassung. Solche Einschränkungen der Kompetenzen der Zentralinstanz der FAR erscheinen eigenartig angesichts der großzügig gewährten, schrankenlosen Erlaubnis zur Intervention bei lokalen »innenpolitischen Unruhen«. Die Diskrepanz läßt keinen $Z_{\text {weifel am }}$ Wesen der Föderation als Notstandsagentur.

Mit der FAR wollten die jeweils herrschenden Militärs neben ihren eigenen Repressionsapparaturen zusätzliche Garantien zur Aufrechterhaltung ihrer Herrschaft erlangen, ohne deshalb ihre Macht durch andere beschneiden zu lassen. Die Jahre der Vereinigung Syriens mit Agypten im Rahmen der Vereinigten Arabischen Republik (1958-1961) haben gezeigt, daß die Union zweier arabischer Länder auf dem Boden nationalistischer Strategien zugleich impliziert, daß die herrschende Gruppe der stärkeren Region ihre Herrschaft auf Kosten der in der schwächeren Region herrschenden Gruppe ausbreitet. Dies war auch der Hintergrund des Putsches der syrischen Offiziere vom 28. September 196r, mit dem sie die Sezession Syriens von der VAR erzwangen ${ }^{15}$. Lehren daraus sind gezogen worden ${ }^{16}$. In der FAR können die syrischen Militärs im Falle eines inneren Aufstandes mit der Hilfe der ägyptischen Armee rechnen, ohne sich deshalb der Herrschaft der ägyptischen Armee beugen zu müssen. Die FAR ist eine Notstandsinstitution, die zur Zementierung der militärischen Herrschaft in Syrien, Libyen und Agypten, aber auch in dem Bruder- und Nichtmitgliedsstaat Sudan beiträgt.

Bassam Tibi

15 Denselben Tatbestand kann man bei den Sezessionskriegen in der Ostregion Nigerias (Biafra) und in Ostpakistan (Bangla Desh) beobachten.

16 Ober diese Zusammenhänge cf. P. Seale, The Struggle for Syria, London I965, sowie M. Kerr, The Arab Cold War, London $1967^{2}$. 